Toм 23 № 3

УДК 512.55

\title{
СООТВЕТСТВИЕ А.И. МАЛЬЦЕВА И ИЗОМОРФИЗМЫ НИЛЬТРЕУГОЛЬНЫХ ПОДКОЛЕЦ АЛГЕБР ШЕВАЛЛЕ ${ }^{1}$
}

\author{
И. Н. Зотов, В. М. Левчук
}

\begin{abstract}
Модели алгебраических систем языка первого порядка называются элементарно эквивалентными пишем $\equiv$, если всякое предложение, истинное в одной из них, является истинным и в другой системе. Теоретико-модельные исследования линейных групп и колец развивались, начиная с работ А.И. Мальцева $(1960,1961$ гг.), в тесной связи с теорией изоморфизмов; как правило, отношение 三 исследуемых систем переносилось на поля (или встречавшиеся кольца) коэффициентов. Соответствие Мальцева исследовалось для колец нильтреугольных матриц и унитреугольных групп (Б. Роуз, 1978 г., В. Вейлер, 1980 г., К. Видэла, 1988 г., О.В. Белеградек, 1999 г., В.М. Левчук, Е.В. Минакова, 2009 г.). Для унипотентных подгрупп групп Шевалле над полем $K$ соответствие исследовал в 1990 г. К. Видэла при char $K \neq 2,3$. Ослабление ограничения на поле $K$ в теореме Видэла авторы анонсировали ранее.
\end{abstract}

В алгебре Шевалле, ассоциированной с системой корней $\Phi$ и полем или кольцом $K$, естественно выделяется нильтреугольная подалгебра $N \Phi(K)$. Основные результаты настоящей статьи устанавливают соответствие Мальцева (взаимосвязано с описанием изоморфизмов) для колец Ли $N \Phi(K)$ классических типов над произвольными ассоциативно коммутативными кольцами с единицей. Отмечается следствие для (неассоциативных) обертывающих алгебр к алгебрам $N \Phi(K)$.

Ключевые слова: алгебра Шевалле, нильтреугольная подалгебра, изоморфизм, теоретико-модельное соответствие Мальцева.

Models of algebraic systems of a first order language are called elementarily equivalent, denoted $\equiv$, if every sentence true in the one is true in the other. The model-theoretic study of linear groups and rings initiated by A.I. Mal'tsev $(1960,1961)$ is closely related to isomorphism theory; as a rule, the relation $\equiv$ of the studied systems was transferred to the fields (or rings encountered) of the coefficients. Maltsev correspondence was investigated for rings of niltriangular matrices and unitriangular groups (B. Rose, 1978, V. Weiler, 1980, K. Videla, 1988, O.V. Belegradek, 1999, V. M. Levchuk, E. V. Minakova, 2009). For unipotent subgroups of Chevalley groups over a field $K$ the correspondence was investigated in 1990 by K. Videla under char $K \neq 2,3$. The weakening of the constraint on the $K$ field in the Videla theorem was announced earlier by the authors.

In the Chevalley algebra associated with the root system $\Phi$ and the field or ring $K$, the niltriangular subalgebra $N \Phi(K)$ is naturally distinguished. The main results of this article establish the Maltsev correspondence (related with the description of isomorphisms) for the Lie rings $N \Phi(K)$ of classical types over arbitrary associative commutative rings with unity. A corollary is noted for (non-associative) enveloping algebras to $N \Phi(K)$.

Keywords: Chevalley algebra, niltriangular subalgebra, isomorphism, Maltsev correspondence.

MSC: 17B30, 17B40, 03C07

\section{Введение}

Классические линейные группы, для которых элементарная эквивалентность переносится на поля или кольца коэффициентов, по-видимому впервые выявлялись в работах А.И. Мальцева [1], [2]. С 1970-х годов теоретико-модельные исследования линейных групп и колец развивались в тесной связи с теорией изоморфизмов, [3]-[5]. Соответствие Мальцева исследовалось для унитреугольных групп и колец нильтреугольных матриц [6]-[10], для групп Шевалле и их унипотентных подгрупп [11], [12].

Алгебру Шевалле над любым полем $K$ характеризуют (см. $\S 1)$ системой корней $\Phi$ и базой Шевалле, включающей элементы $e_{r}(r \in \Phi),[13, \S 4.4]$. Подалгебру $N \Phi(K)$ с базой $\left\{e_{r} \mid r \in \Phi^{+}\right\}$

\footnotetext{
${ }^{1}$ Работа выполнена при поддержке РФФИ (проект 16-01-007-07).
} 
называют нильтреугольной. В обзоре [14] отмечается вопрос о соответствии Мальцева для колец Ли $N \Phi(K)$ над ассоциативно коммутативными кольцами $K$ с единицей. В настоящей статье этот вопрос решается для классических типов взаимосвязано с описанием изоморфизмов.

Основные теоремы об изоморфизмах и соответствии Мальцева приведены в следующем параграфе (теоремы 1 и 2). Вспомогательный $\S 2$ посвящен автоморфизмам и характеристическим идеалам колец Ли $N \Phi(K)$. Теорема об изоморфизмах колец Ли $N \Phi(K)$ доказывается в $\S 3$, а теорема о соответствии Мальцева - в $~ 4$; там же отмечается следствие для (неассоциативных) обертывающих алгебр к алгебрам Ли $N \Phi(K)$.

\section{1. Предварительные замечания и основные теоремы}

Модели алгебраических систем $\mathscr{U}$ и $\mathscr{U}^{\prime}$ языка первого порядка $\mathscr{L}$ называются элементарно эквивалентными - пишем $\mathscr{U} \equiv \mathscr{U}^{\prime}$, если всякое предложение, истинное в одной из них, является истинным и в другой системе. Известна изоморфность таких алгебраических систем, когда они равномощны, но в общем случае это не так. Например, поле комплексных чисел элементарно эквивалентно полю алгебраических чисел, однако, эти поля не изоморфны, поскольку их мощности различны, [3].

Алгебру Шевалле $\mathcal{L}_{\Phi}(K)$ ассоциируют с любыми полем $K$ и системой $\Phi$, характеризуя базой Шевалле $\left\{e_{r}(r \in \Phi), h_{s}(s \in \Pi)\right\}$, где П - система простых корней или база в $\Phi$. Известно, что $p(\Phi):=\max \{(r, r) /(s, s) \mid r, s \in \Phi\}=1,2$ или (тип $\left.G_{2}\right) 3$.

Структурные константы базы Шевалле целочисленные. Более точно, если $r, s \in \Phi$, то [13]:

$$
\begin{gathered}
e_{r} * e_{-r}=h_{r}, \quad h_{s} * h_{r}=0, \quad h_{s} * e_{r}=\frac{2(r, s)}{(r, r)} e_{r} ; \\
e_{r} * e_{s}=0 \quad(r+s \notin \Phi \cup\{0\}), \quad e_{r} * e_{s}=N_{r s} e_{r+s}=-e_{s} * e_{r} \quad(r+s \in \Phi),
\end{gathered}
$$

где $N_{r s}= \pm 1$, или $|r|=|s|<|r+s|$ и $N_{r s}= \pm 2$, или (тип $\left.G_{2}\right) N_{r s}= \pm 2$ или \pm 3 . Произвол в выборе знаков констант $N_{r s}$ описан в [13, Предложение 4.2.2].

Система положительных корней $\Phi^{+} \supseteq$ П в $\Phi$ единственна. Элементы $e_{r}\left(r \in \Phi^{+}\right)$образуют базу нильтреугольной подалгебры $N \Phi(K)$ алгебры Шевалле.

Для любого корня $r$ отображение $t \rightarrow x_{r}(t):=\exp \left(t \cdot a d e_{r}\right)(t \in K)$ дает изоморфизм аддитивной группы поля $K$ в группу автоморфизмов Aut $\mathcal{L}_{\Phi}(K)$. Группу Шевалле $\Phi(K)$ порождают корневые подгруппы $X_{r}=x_{r}(K)$. Её унипотентную подгруппу $U \Phi(K)$ порождают корневые подгруппы $X_{r}\left(r \in \Phi^{+}\right)$, [13].

Алгебра $\mathcal{L}_{\Phi}(K)$ и группа Шевалле $\Phi(K) \subseteq A u t \mathcal{L}_{\Phi}(K)$ естественно определены, как и выше, над любым ассоциативно коммутативным кольцом $K$ с единицей. Изоморфизм $\theta: K \rightarrow S$ колец коэффицентов, очевидно, всегда индуцирует изоморфизм колец Ли $\bar{\theta}: N \Phi(K) \rightarrow N \Phi(S)$ по правилу $\bar{\theta}: x e_{r} \rightarrow \theta(x) e_{r} \quad(x \in K, r \in \Phi)$.

Биективное отображение $\tau: \Phi \rightarrow \Phi^{\prime}$ систем корней называют их эквивалентностъю, если существует вещественное число $\lambda>0$ такое, что $(\tau(r), \tau(s))=\lambda \cdot(r, s) \quad(r, s \in \Phi)$. Ясно, что любая эквивалентность $\tau$ индуцирует изоморфизм $\bar{\tau}$ алгебр Ли по правилу

$$
\bar{\tau}: N \Phi(K) \rightarrow N \Phi^{\prime}(K), \quad e_{r} \rightarrow e_{\tau(r)}\left(r \in \Phi^{+}\right) .
$$

Далее (если не оговорено противное), $K$ и $S$ - произвольные ассоциативно коммутативные кольца с единицами. Основной теоремой об изоморфизмах в статье является

Теорема 1. Пусть $N \Phi(K)$ - кольцо Ли классического типа $D_{n}(n \geq 4), B_{n}$ или $C_{n}(n>$ 4). Кольио Ли $N \Phi^{\prime}(S)$ изоморфно $N \Phi(K)$ тогда и только тогда, когда $S \simeq K$, а системь корней $\Phi^{\prime}$ и $\Phi$ эквивалентны. 
3 а м е ч а н и е 1 . Хорошо известны тесные структурные связи кольца Ли $N \Phi(K)$ и унипотентной группы $U \Phi(K)$. Так, в силу [15], когда $\Phi$ типа $A_{n}(n>2)$, имеем:

$$
U \Phi(K) \simeq U \Phi^{\prime}(S) \Longleftrightarrow N \Phi(K) \simeq N \Phi^{\prime}(S) .
$$

См. также соответствие в [16] между нормальными подгруппами в $U \Phi(K)$ и идеалами кольца Ли $N \Phi(K)$. По теореме 1 алгебры Ли $N \Phi(K)$ типа $B_{n}$ и $C_{n}$ при $n>2$ всегда не изоморфны, хотя [13] группы Шевалле (аналогично, унипотентные подгруппы) типа $B_{n}$ и $C_{n}$ над совершенным полем или кольцом характеристики 2 изоморфны.

Выберем кольцо Ли $N \Phi(K)$ как и в теореме 1. Соответствие Мальцева выявляет

Теорема 2. Кольца Ли $N \Phi^{\prime}(S)$ и $N \Phi(K)$ элементарно эквивалентны в том и только в том случае, когда $K \equiv S$, а системы корней $\Phi$ и $\Phi^{\prime}$ эквивалентны.

Используемые терминология и обозначения, связанные с системами корней, алгебрами и группами Шевалле, стандартны и соответствуют, главным образом, [13].

\section{2. Автоморфизмы и характеристические идеалы}

Очевидна

Лемма 1. Пусть $\psi: R \rightarrow R^{\prime}$ - изоморфизм произвольных колеи, $R$ u $R^{\prime}, \pi \in$ Aut $R u$ $\chi \in$ Aut $R^{\prime}$. Тогда $\pi \psi \chi$ есть также изоморфизм кольиа $R$ на $R^{\prime}$.

Как и в [17], мы используем известное обобщение центральных автоморфизмов, то есть тождественных по модулю центра. Автоморфизм группы или кольца Ли $R$, являющийся единичным по модулю $m$-го гиперцентра и внешним автоморфизмом по модулю $(m-1)$-го гиперцентра, называют гиперцентральным высоты $m$ или, кратко, гиперцентральным автомор$\oint и з м о м$, когда $R$ не совпадает с $m$-м гиперцентром.

Нам потребуются некоторые характеристические идеалы и автоморфизмы кольца Ли $N \Phi(K)$.

Гиперцентры $Z_{j}=Z_{j}(K)$, то есть члены гиперцентрального (или верхнего центрального) ряда и члены $\Gamma_{i}$ нижнего центрального ряда кольца Ли $N \Phi(K)$, а также их централизаторы являются его характеристическими идеалами.

Bысотой корня $r$ называют сумму $h t(r)$ коэффициентов в разложении $r$ по базе П. Число Кокстера $h=h(\Phi)$ системы $\Phi$ равно $h t(\rho)+1$ с максимальным в $\Phi^{+}$корнем $\rho$. Идеалы $L_{m}$ с базой $\left\{e_{r} \mid r \in \Phi^{+}, h t(r) \geq m\right\}$ образуют центральный ряд $L_{1} \supset L_{2} \supset \cdots \supset L_{h}=0$ в $N \Phi(K)$, называемый стандартным. Аналогично [17, Лемма 1], индукцией по $h-i$ доказывается формула $\Gamma_{i}=L_{i}=Z_{h-i} \quad(1 \leq i \leq h)$. Поэтому справедлива

Лемма 2. Верхний и нижний центральные рядъ кольца Ли $N \Phi(K)$ при $p(\Phi) ! K=K$ совпадают с её стандартным иентральным рядом: $\Gamma_{i}=L_{i}=Z_{h-i}(1 \leq i \leq h)$.

Отметим, что ограничение на кольцо $K$ в лемме равносильно требованию $2 K=K$, когда $\Phi$ типа $B_{n}, C_{n}$ и $F_{4}$, и $6 K=K$ для типа $G_{2}$. В остальных случаях все корни в $\Phi$ одной длины и, в силу леммы, все идеалы $L_{m}$ кольца Ли $N \Phi(K)$ характеристические.

Пусть $\{r\}^{+}-$множество корней $s \in \Phi^{+}$таких, что в разложении $s-r$ по базе П все коэффициенты неотрицательны. В алгебре $N \Phi(K)$ подалгебры с базисом $\left\{e_{s} \mid s \in\{r\}^{+}\right\}$и $\left\{e_{s} \mid s \in\{r\}^{+}, s \neq r\right\}$ есть идеалы, обозначаемые через $T(r)$ и $Q(r)$, соответственно.

Обычные матричные единицы $e_{i j}(1 \leq j<i \leq n)$ составляют базу алгебры $N T(n, K)$ нижних нильтреугольных $n \times n$ матриц (с нулями на главной диагонали и над ней) над $K$. Ассоциированная к ней алгебра Ли представляет алгебру $N \Phi(K)$ типа $A_{n-1}$ с базой Шевалле 
$\left\{e_{r} \mid r \in \Phi^{+}, e_{r}=e_{i j}\right\}$ после соответствующей нумерации корней $r=r_{i j}$. Изоморфизмы колец Ли $N \Phi(K)$ в этом случае известны, [15].

Алгебры Ли $N \Phi(K)$ типа $B_{n}, C_{n}$ и $D_{n}$ заданы в [17] также в базе из "матричных единиц" $e_{i v}$ с ограничениями, соответственно,

$$
-i<v<i \leq n, \quad-i \leq v<i \leq n, v \neq 0, \quad 1 \leq|v|<i \leq n .
$$

Суммы двух корней, являющиеся корнем, помимо стандартных $r_{i j}+r_{j v}=r_{i v}$ здесь еще

$$
r_{k v}+r_{m,-v}=r_{k,-m} \quad(k>m>|v|),
$$

а для типа $C_{n}$ также $r_{k v}+r_{k,-v}=r_{k,-k}(k>|v|)$. Согласно [17, Лемма 2], справедлива

Лемма 3. Знаки структурных констант базиса Шевалле можно выбрать так, что е $e_{j v}=e_{i v}$ и верны равенства:

$$
\begin{gathered}
\Phi=B_{n}, D_{n}: \quad e_{j v} * e_{i,-v}=e_{i,-j} \quad(i>j>|v|>0) ; \\
\Phi=B_{n}: \quad e_{i 0} * e_{j 0}=2 e_{i,-j} \quad(i>j) ; \\
\Phi=C_{n}: e_{j m} * e_{i,-m}=e_{i m} * e_{j,-m}=e_{i,-j}(i>j>m \geq 1), \quad e_{i j} * e_{i,-j}=2 e_{i,-i}(i>j \geq 1) .
\end{gathered}
$$

Всякий элемент алгебры Ли $N \Phi(K)$ любого классического типа представляется $\Phi^{+}$-матрицей ||$a_{i v} \mid=\sum a_{i v} e_{i v}$ соответствующего типа. Так, $B_{n}^{+}$- матрицы имеют вид

$$
\begin{aligned}
& a_{10} \\
& \begin{array}{lll}
a_{2,-1} & a_{20} & a_{21}
\end{array} \\
& \text { ․ } \quad \ldots \quad \ldots \\
& \begin{array}{llllllll}
a_{n,-n+1} & \ldots & a_{n,-1} & a_{n 0} & a_{n 1} & \ldots & a_{n, n-1} .
\end{array}
\end{aligned}
$$

$D_{n}^{+}$-матрицы получаем, отбрасывая 0-й столбец; они дают подалгебру в $N B_{n}(K)$. В алгебре Ли $N B_{n}(K)$ (или $N \Phi(K)$ типа $B_{n}$ ) выделяем подмодули $R_{j}:=\sum_{i=j}^{n} K e_{i 0}, 1 \leq j \leq n$, а также подмодуль $L_{j}^{[0}$ с базой $\left\{e_{u v} \mid 0 \leq v<u \leq n, u-v \geq j\right\}$.

Пусть $T_{i m}$ - идеал всех $\Phi^{+}$-матриц $\left\|a_{u v}\right\|$ с условием $a_{u v}=0$ при $u<i$ или $v>m$. Ясно, что $T_{i m}=T(r)$ при определенном $r=r_{i m} \in \Phi^{+}$, кроме $T_{i 1}=T\left(r_{i,-1}\right)+T\left({ }_{i, 1}\right)$ в случае $m=1$ для типа $D_{n}$. Считаем $T_{1 m}:=T_{i m}$, если при выбранных $\Phi$ и $m$ номер $i$ - наименьший.

Используя лемму 3 , сейчас несложно выписать централизаторы $C\left(T_{i m}\right)$. Пусть $\mathcal{A}_{2}:=A n n_{K}(2)$ - аннулятор элемента 2 в кольце $K$.

Лемма 4. Для алгебр Ли $N \Phi(K)$ при $i<n$ выполняются следующие равенства.

$$
\begin{gathered}
\Phi=D_{n}: \quad C\left(T_{i m}\right)=T_{1,-m-1} \quad(0<|m|<i) . \\
\Phi=B_{n}: C\left(T_{i m}\right)=T_{1,-m-1} \quad(-i<m<0), \quad C\left(T_{i m}\right)=T_{1,-m-1}+\mathcal{A}_{2} \cdot R_{m+1} \quad(0 \leq m<i) . \\
\Phi=C_{n}: \quad C\left(T_{i m}\right)=T_{1,-m-1} \quad(-i \leq m<i) .
\end{gathered}
$$

Равенства будут верны и при $i=n$, если в правых частях прибавить $\mathcal{A}_{2} T_{n n-1}$ для типа $C_{n}$, $u T_{n n-1}$ для типов $B_{n}$ и $D_{n}$.

Леммы 3 и 4 легко завершают описание центральных рядов алгебр Ли $N \Phi(K)$ классических типов. Исключительные в лемме 2 гиперцентральные ряды для типов $B_{n}$ и $C_{n}$ дает 
Лемма 5. Гипериентры алгебры Ли $N C_{n}(K)(n \geq 2)$ записываются в виде

$$
Z_{i}=L_{2 n-i}+\mathcal{A}_{2} L_{2 n-i-1}(1 \leq i<2 n-1), \quad Z_{2 n-1}=L_{1} .
$$

Для алгебры $N B_{n}(K)(n \geq 2)$ имеем:

$$
\begin{aligned}
& Z_{i}=L_{2 n-i}+\mathcal{A}_{2} R_{n+1-i}(1 \leq i \leq n-2), \quad Z_{n-1}=L_{n+1}+\mathcal{A}_{2} R_{2}+\mathcal{A}_{2} e_{n 1} \\
& Z_{n+i}=L_{n-i}+\mathcal{A}_{2} R_{1}+\mathcal{A}_{2} L_{n-i-2}^{[0} \quad(0 \leq i \leq n-3), \quad Z_{2 n-2}=L_{2}+\mathcal{A}_{2} L_{1} .
\end{aligned}
$$

Лемма 6. Пусть $2 K \neq K$ u $n \geq 2$. Тогда кольца Ли $N B_{n}(K)$ u $N C_{n}(K)$ порождают

$$
\left\{K e_{i i-1}(1 \leq i \leq n) ; \quad K e_{2,-1}\right\}, \quad\left\{K e_{i i-1}(2 \leq i \leq n) ; \quad K e_{i,-i}(1 \leq i \leq n)\right\},
$$

соответственно, и ни одно $K e_{i v}$ в них нельзя отбросить. Если $n \geq 3$, то коммутант содержит центр в кольще $N B_{n}(K)$, а в $N C_{n}(K)$ - нет, и $N B_{n}(K) \nsucceq N C_{n}(K)$.

Д о к а з а т е л ь с т в о. При $n>2$ максимальный корень системы корней типа $B_{n}$ представляется суммой двух длинных корней и в кольце Ли $N B_{n}(K)(n>2)$ имеем:

$$
\Gamma_{i}=L_{i}^{[0}+L_{i+2}+2 L_{i}(2 \leq i \leq n), \quad \Gamma_{2} \supset T_{31} \supset K e_{n,-n+1}+\mathcal{A}_{2} e_{n 0}=Z_{1} .
$$

Для алгебры Ли $N C_{n}(K)$ обозначим через $T_{0}$ подмодуль с базой из элементов $e_{i v}$ с условием $|v|<i$, то есть соответствующие, в точности, всем коротким корням. Учитывая, что корень $r_{t,-t}$ записывается суммой двух корней лишь в виде $r_{t,-t}=r_{t m}+r_{t,-m}$, находим, помимо центра $Z_{1}=K e_{n,-n}+\mathcal{A}_{2} e_{n,-n+1}$, члены нижнего центрального ряда.

$$
\Gamma_{1}=L_{1}, \quad \Gamma_{i}:=L_{1} * \Gamma_{i-1}=\left(L_{i} \cap T_{0}\right)+\sum_{i / 2<t \leq n} 2 K e_{t,-t} \quad(1<i<2 n) .
$$

При $2 K \neq K$ центр $Z_{1}$ не лежит в $T_{0}+\sum_{1<t \leq n} 2 K e_{t,-t}$ и, тем более, в коммутанте $\Gamma_{2}$. Тем самым, второе утверждение леммы доказано; первое - очевидно. Лемма доказана.

Стандартными автоморфизмами кольца Ли $N \Phi(K)$ считаем произведения кольцевых, внутренних (или из $U \Phi(K)$ ), диагональных и центральных автоморфизмов.

Выделим некоторые нестандартные автоморфизмы. Пусть $\Pi=\left\{q, r_{1}, r_{2}, r_{3}\right\}$ для типа $D_{4}$, где $q$ - простой корень, неподвижный относительно всех симметрий графа Кокстера. Тогда, в силу [18], любой матрице $\beta=\left\|b_{u v}\right\| \in S L(3, K)$ с условиями $2 b_{m j} b_{m i}=0,1 \leq i, j, m \leq 3, i \neq j$, соответствует автоморфизм $\widehat{\beta}$ алгебры $N D_{4}(K)$, определяемый действием

$$
\widehat{\beta}: e_{r_{i}} \rightarrow \sum_{m=1}^{3} b_{i m} e_{r_{m}}(i=1,2,3), \quad e_{q} \rightarrow e_{q} .
$$

Для простых симметричных корней $r$ и $\bar{r} \neq r(\overline{\bar{r}}=r)$ системы корней $\Phi$ типа $D_{n}(n \geq 4)$, согласно [19], определено изоморфное вложение ${ }^{\sim}$ подгруппы

$$
S=\left\{\alpha=\left\|a_{u v}\right\| \in S L(2, K): 2 a_{11} a_{12}=2 a_{21} a_{22}=0\right\}
$$

группы $S L(2, K)$ в группу автоморфизмов алгебры Ли $N \Phi(K)$ по правилу

$$
\widetilde{\alpha}: \quad e_{r} \rightarrow a_{11} e_{r}+a_{12} e_{\bar{r}}, \quad e_{\bar{r}} \rightarrow a_{21} e_{r}+a_{22} e_{\bar{r}}, \quad e_{s} \rightarrow e_{s} \quad(s \in \Pi \backslash\{r, \bar{r}\}) .
$$

В матричном представлении алгебры $N D_{n}(K)$ он определяется правилом

$$
\widetilde{\alpha}: e_{2,-1} \rightarrow a_{11} e_{2,-1}+a_{12} e_{21}, \quad e_{21} \rightarrow a_{21} e_{2,-1}+a_{22} e_{21}, \quad e_{i+1 i} \rightarrow e_{i+1 i}(1<i<n) .
$$


При обратимых $1+t \in 1+\mathcal{A}_{2}$ для типа $B_{n}$ выделяем полудиагональные автоморфизмы

$$
\delta_{t}^{(-1)}: e_{k v} \rightarrow(1+t) e_{k v}(0<-v<k \leq n), \quad e_{k v} \rightarrow e_{k v}(0 \leq v<k \leq n) .
$$

Автоморфизмы колец Ли $N \Phi(K)$ исследовались ранее [18], наряду с описаниями групп автоморфизмов Aut $U \Phi(K)$. К.Видэла [11] использует нестандартный (extremal) aвтоморфизм Гиббса [20] группы $U \Phi(K)$ при $K=6 K$; аналогичный автоморфизм алгебр Ли $N \Phi(K)$ см. [21]. Согласно [17], эти автоморфизмы - гиперцентральные высоты 3 (тип $C_{n}$ ) или 2. См. также описание автоморфизмов алгебр Ли $N \Phi(K)$ при $K=2 K$ в [21].

Подгруппу автоморфизмов алгебры Ли $N \Phi(K)$, которую порождают выделенные в [19] гиперцентральные автоморфизмы высоты $>1$, обозначаем через $V(\Phi, K)$. Так, каждому элементу $t \in \mathcal{A}_{2}$ в [19] сопоставлены гиперцентральные автоморфизмы

$$
\alpha=\left\|a_{u v}\right\| \rightarrow \alpha+t\left(a_{n n-1} e_{n-2,-n+3}+a_{n n-2} e_{n-1,-n+3}+a_{n n-3} e_{n-1,-n+2}\right),
$$

алгебр Ли $N D_{n}(K)(n \geq 5), N B_{n}(K)$ и $N C_{n}(K)(n \geq 4)$, а также

$$
\alpha \rightarrow \alpha+t\left(a_{n n-1} e_{n-2,0}+a_{n n-2} e_{n-1,0}\right), \quad \chi_{t}: \alpha \rightarrow \alpha+\sum_{k=2}^{n-1} a_{k,-1} t e_{k 0}
$$

алгебры Ли $N B_{n}(K)(n \geq 4)$ высоты $\leq 3$ и $\leq n-1$, соответственно, по лемме 5. Описание автоморфизмов колец Ли $N \Phi(K)$ классических типов завершено в [19]. Его резюмирует

Теорема 3. Всякий автоморфизм кольца Ли $N C_{n}(K), n>4$, есть произведение стандартного и гиперцентрального из $V(\Phi, K)$ автоморбизмов. Для кольца Ли $N B_{n}(K), n>4$, сомножителем добавляется полудиагональный автоморфизм, а для кольца Ли $N D_{n}(K)-$ автоморбизм из $\widetilde{S}$ при $n \geq 5$ или (2.1) $п р и ~ n=4$.

3 а м е ч а н и е 2. Описание в [19] подгруппы $V(\Phi, K)$ для классических типов показывает, что идеал $T_{10}$ кольца Ли $N B_{n}(K)$ характеристичен при $n>4$, а в кольце Ли $N C_{n}(K)(n>4)$ идеал $T_{i v}$ при $i<n$ - характеристический. Идеалы $T_{n n-1}, T_{n n-2}, T_{n n-3}$ являются $V(\Phi, K)$ инвариантными по модулю $T_{n-2,-n+3}$ в кольцах Ли $N D_{n}(K)(n \geq 4)$ и $N C_{n}(K)(n>4)$, а по модулю $T_{n-2,-n+3}+T_{n-2,0}-$ в кольце $N B_{n}(K)(n>4)$.

\section{3. Теорема об изоморфизмах}

Доказательство теоремы 1 будет опираться на две следующие леммы.

Лемма 7. Пусть кольиа Ли $N \Phi(K)$ и $N \Phi^{\prime}(S)$ изоморфны, $\Phi$ ранга $>1$, причем $2 K=K$ для типа $F_{4}$ и $6 K=K$ для типа $G_{2}$. Тогда системы корней $\Phi$ и $\Phi^{\prime}$ эквивалентны.

Д о к а з а т е л ь с т в о. Допустим, что кольца Ли $N \Phi(K)$ и $N \Phi^{\prime}(S)$ изоморфны, то есть существует изоморфизм $\phi: N \Phi(K) \rightarrow N \Phi^{\prime}(S)$.

Пусть $r, s \in \Pi$ и $r+s \in \Phi^{+}$. Так как $r-s \notin \Phi$, то $e_{r} * e_{s}=N_{r s} e_{r+s}= \pm e_{r+s}$. Кроме того, $\Phi(r, s):=(Z r+Z s) \cap \Phi-$ подсистема корней ранга 2 в $\Phi$, причем подсистема типа $G_{2}$ встречается лишь для $\Phi$ типа $G_{2}$. В графе Кокстера системы $\Phi$ корням $r$ и $s$ соответствуют соседние вершины. Если граф Кокстера системы $\Phi$ имеет более двух крайних корней, то $\Phi(r, s)$ типа $A_{2}$ и $\Phi$ типа $D_{n}$ или $E_{n}$. Система $\Phi$ типа $D_{n}$ отделяется от систем $\Phi$ типа $E_{n}$ тем, что в ней есть корень соседний с тремя другими, два из которых крайние. Системы $\Phi$ типа $E_{n}(n=6,7,8)$ различаются числами Кокстера $h=h(\Phi)$, определяющими ступень нильпотентности алгебры $N \Phi(K)$.

В системе корней $\Phi$ типа $A_{n}$ нет корней соседних с тремя другими и $\Phi(r, s)$ всегда типа $A_{2}$; в этом случае изоморфизмы изучены в [15]. В оставшихся трёх системах корней встречается 
подсистема корней $\Phi(r, s)$ типа $B_{2}$. Заметим, что в системах корней типа $B_{n}$ и $C_{n}$ любая подсистема корней ранга 4 имеет число Кокстера $\leq 8$. Поэтому система корней $\Phi$ типа $F_{4}$ отделяется от них, поскольку её ранг 4 и число Кокстера равно 12.

Графы Кокстера систем корней $\Phi$ типов $B_{n}$ и $C_{n}$ совпадают, но при $n>2$ они различаются схемами Дынкина

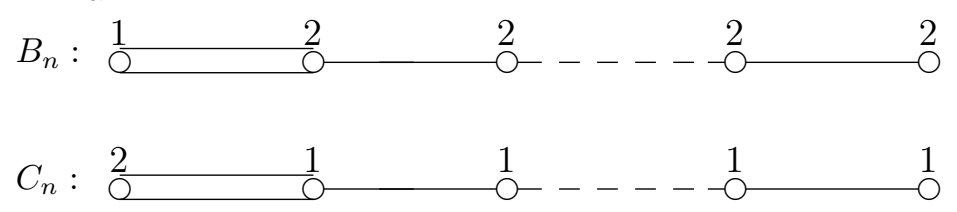

(см. также [16], [22]). В силу леммы 6 , при $2 K \neq K$ также имеем $N B_{n}(K) \not N C_{n}(K)$.

По лемме 2 , если в $\Phi$ все корни одной длины (равносильно, все подсистемы $\Phi(r, s)$ - типа $\left.A_{2}\right)$ или $\Phi$ - одного из типов $B_{n}, C_{n}, F_{4}$ и $2 K=K$, то $L_{i}=L_{i}(N \Phi(K))$ - характеристические идеалы кольца Ли $N \Phi(K)$ и поэтому

$$
\phi\left(L_{i}(N \Phi(K))\right)=L_{i}\left(N \Phi^{\prime}(S)\right) \quad(1 \leq i<h(\Phi)) .
$$

Итак, мы можем установить биективное отображение баз $\tau: \Pi(\Phi) \rightarrow \Pi\left(\Phi^{\prime}\right)$, соответствующее выбранным схемам Дынкина. Отображение $\tau$ продолжается на произвольный корень $r=\sum_{p \in \Pi(\Phi)} c_{p} p \in \Phi^{+}$по правилу

$$
\tau(r)=\sum_{p \in \Pi(\Phi)} c_{p} \tau(p) \in \Phi^{+} .
$$

Отсюда получаем изоморфизм $e_{r} \rightarrow e_{\tau(r)} ;\left(r \in \Phi^{+}\right)$алгебр Ли $N \Phi(K)$ и $N \Phi^{\prime}(S)$, индуцированный эквивалентностью систем корней. Лемма доказана.

Как следствие, вопрос об изоморфизмах $N \Phi(K) \rightarrow N \Phi^{\prime}(S)$ сводится доказанной леммой к вопросу об изоморфизмах $N \Phi(K) \rightarrow N \Phi(S)$.

Лемма 8. Всякий изоморфизм $\phi: N \Phi(S) \rightarrow N \Phi(K)$ колеи Ли классического типа ранга $n>3$ над ассочиативно коммутативными кольцами $S$ и $K$ с единицами есть произведение $\phi=\eta \bar{\theta}$ для подходящего изоморфизма $\theta: S \rightarrow K u \eta \in$ Aut $N \Phi(S)$.

Д о к а з а т е л ь с т в о. Для простого корня $r$ идеал $T(r)$ алгебры $N \Phi(K)$ типа $A_{n}$ всегда является (максимальным) абелевым. По лемме 3 , для других классических типов абелевость $T(r)$ выполняется лишь когда $r$ соответствует в графе Кокстера какой-либо из крайних вершин, причем для типа $D_{n}$ любой из них.

Отметим, что $\phi$-образ идеала $L_{i}(S)$ кольца Ли $N D_{n}(S)$ совпадает с идеалом $L_{i}(K)$ кольца Ли $N D_{n}(K)$, причем $N \Phi(S) / L_{i}(S) \rightarrow N \Phi(K) / L_{i}(K)$ - изоморфизм фактор-колец. С точностью до умножения $\phi$ на автоморфизм из $\widetilde{S}$ при $n \geq 5$ или вида (2.1) при $n=4$, в силу теоремы 3 и леммы 1 , существуют изоморфизмы $\theta_{a}(a \in \Pi)$ аддитивной группы $(S,+)$ на $(K,+)$ с условием $\phi\left(x e_{a}\right)=\theta_{a}(x) e_{a} \bmod L_{2}$ и при $x, y \in S, b \in \Pi, a+b \in \Phi$ имеем

$$
\theta_{a}(S)=\theta_{b}(S)=K, \quad \phi\left(x e_{a} * y e_{b}\right)=\phi\left(x e_{a}\right) * \phi\left(y e_{b}\right)=\theta_{a}(x) \theta_{b}(y) e_{a+b} \quad \bmod L_{3}(K) .
$$

Так как $\theta_{a}\left(1_{S}\right) K=K=\theta_{b}\left(1_{S}\right) K$, то $\theta_{a}\left(1_{S}\right)$ - обратимый элемент кольца $K$ при любом $a \in \Pi$. $\mathrm{C}$ точностью до умножения $\phi$ на диагональный автоморфизм, можем считать $\theta_{a}\left(1_{S}\right)=1_{K}$ для всех $a \in \Pi$. Отсюда легко приходим к равенствам $\theta_{a}=\theta_{b}$ для любых $a, b \in \Pi$. Полагая $\theta=\theta_{a}$, получаем, что $\theta: S \rightarrow K$ - кольцевой изоморфизм и лемма доказана для типа $D_{n}$.

В силу замечания 2 , для кольца Ли $N C_{n}(K)(n>4)$ идеалы $T_{i v}(K)$ при $i<n$ являются характеристичными. Так как $\phi\left(T_{i v}(S)\right)=T_{i v}(K), i<n$, то $\phi$ индуцирует изоморфизм факторкольца $N C_{n}(S) / T_{2,-2}(S)$ на кольцо $N A_{n}(K) \simeq N T(n+1, K)$. Учитывая описание Aut $N T(n, K)$ $\left[23\right.$, Теоремы 1 и 2] и изоморфизмов [15], получаем утверждение леммы для типа $C_{n}$. 
В кольце Ли $N B_{n}(K)(n>4)$ имеем $\phi\left(T_{10}(S)\right)=T_{10}(K)$, в силу замечания 2 . Поэтому $\phi$ индуцирует изоморфизм фактор-кольца $N B_{n}(S) / T_{10}(S)$ на $N A_{n-1}(K) \simeq N T(n, K)$ и утверждение леммы завершаем аналогично типу $C_{n}$. Лемма доказана.

Применяя леммы 7 и 8, легко получаем утверждение теоремы 1.

В заключении параграфа отметим, что подгруппа центральных автоморфизмов кольца Ли шире, чем для алгебры Ли, например, [23]. Кроме того, если $\theta \in A u t K$, то автоморфизм $\bar{\theta}$ кольца Ли $N \Phi(K)$ является автоморфизмом алгебры Ли $N \Phi(K)$ лишь при $\theta=1$.

\section{4. Соответствие Мальцева}

В этом параграфе доказывается теорема 2 о соответствии Мальцева для нильтреугольных колец $N \Phi(K)$ классических типов. Принципиальным является переход от элементарной эквивалентности к изоморфизму соответствующих систем. Известна

Теорема 4. [3, Теорема 6.1.15] Алгебраические системъ $\mathfrak{A}$ и $\mathfrak{B}$ элементарно эквивалентны тогда и только тогда, когда существуют их изоморфные ультрастепени.

В [3] также приведены понятия фильтра, фильтрованного произведения, ультрапроизведения и ультрастепени. Аналогично [24, Лемма 2.3] выявляется связь ультрастепеней алгебраической системы с алгебраической системой над ультрастепенями.

Лемма 9. Для любого ассоциативно коммутативного кольца $K$ с единищей и ультрафильтра $(D, I)$ существует естественный изоморфизм $N \Phi(K)^{I} / D \simeq N \Phi\left(K^{I} / D\right)$.

Завершим сейчас доказательство теоремы 2.

Если $K \equiv S$, то элементарная эквивалентность колец Ли $N \Phi(S) \equiv N \Phi(K)$ доказывается стандартными методами, см. [9] и [24, Лемма 2.5].

Допустим далее, что $N \Phi^{\prime}(S) \equiv N \Phi(K)$. По теореме 4 , существует ультрафильтр $(D, I)$ такой, что $N \Phi^{\prime}(S)^{I} / D \simeq N \Phi(K)^{I} / D$.

Используя лемму 9 , получаем

$$
N \Phi^{\prime}(S)^{I} / D \simeq N \Phi^{\prime}\left(S^{I} / D\right), \quad N \Phi(K)^{I} / D \simeq N \Phi\left(K^{I} / D\right),
$$

откуда

$$
N \Phi^{\prime}\left(S^{I} / D\right) \simeq N \Phi\left(K^{I} / D\right) .
$$

В силу теоремы 1 находим изоморфизм $K^{I} / D \simeq S^{I} / D$ и поэтому $K \equiv S$ по теореме 4 .

Тем самым, доказательство теоремы 2 завершается.

3 а м е ч а н и е 3. Алгебра $A=(A,+, \cdot)$ (не обязательно ассоциативная) названа в [25] обертывающей алгебры Ли $L$, если алгебра $A^{(-)}=(A,+, *)$ с новым умножением $a * b:=$ $a b-b a$ (коммутирование) изоморфна $L$; обе алгебры можем построить на одном линейном пространстве. Автоморфизмы и изоморфизмы алгебры $A$ есть соответственно автоморфизмы и изоморфизмы алгебры Ли $L$, поскольку основные операции в $L$ производны от операций в $A$. Поэтому из доказанной теоремы 2 следует, что соответствие Мальцева выполняется и для построенных в [25] обертывающих алгебр нильтреугольных алгебр $N \Phi(K)$ классических типов. 


\section{СПИСОК ЛИТЕРАТУРЫ}

1. Мальцев А.И. Об одном соответствии между кольцами и группами // Матем. сборник. 1960. Т. 50. С. 257-266. // Мат. сб. 1960. Т. 50, № 2. С. 3-12.

2. Мальцев А.И. Элементарные свойства линейных групп // Некоторые проблемы в Математике и механике / Издательство АН СССР. Новосибирск. 1961. С. 110-132.

3. Кейслер Г., Чэн Ч.Ч. Теория моделей. М.: Мир, 1977. 614 с.

4. Hodges W. Model Theory. - Cambridge: Cambridge Univ. Press, 1993. 772 p.

5. Ремесленников В.Н., Романьков В.А. Теоретико-модельные и алгоритмические вопросы теории групп // Итоги науки и техники. Серия: Алгебра, топология, геометрия. 1983. Т. 21. С. 3-79.

6. Rose B.I. The $\chi_{1}$-categoricity of Strictly Upper Triangular Matrix Rings over Algebraically Closed Fields // J. Symbolic Logic. 1978. Vol. 43, № 2. P. 250-259.

7. Wheeler W.H. Model Theory of strictly upper triangular matrix ring // J. Symbolic Logic. 1980. Vol. 45. P. $455-463$.

8. Videla C.R. On the Model Theory of the Ring $N T(n, R) / /$ Pure and Appl. Algebra. 1988. Vol. 55. P. 289-302.

9. Belegradek O.V. Model Theory of Unitriangular Groups // Amer. Math. Soc. Transl. 1999. Vol. 195, № $2.115 \mathrm{p}$.

10. Левчук В.М., Минакова Е.В. Элементарная эквивалентность и изоморфизмы локальнонильпотентных матричных групп и колец // Доклады РАН. 2009. Т.425, № 2. С. 165-168.

11. Videla C.R. On the Mal'cev correspondence // Proceed. AMS. 1990. V. 109. № 2. P. 493-502.

12. Бунина Е.И., Михалев А.В., Пинус А.Г. Элементарная и близкая к ней логические эквивалентности классических и универсальных алгебр. М: МЦНМО, 2015. 360 с.

13. Carter R.W. Simple groups of Lie type. New York: Wiley and Sons, 1972. 331 p.

14. Левчук B.M. Теоретико-модельные и структурные вопросы алгебр и групп Шевалле // Итоги науки. Юг России. Т. 6. Группы и графы. Владикавказ: ЮМИ ВНЦ РАН и РСО-А, 2012. С. 71-80.

15. Kuzucuoglu F., Levchuk V.M. Isomorphisms of Certain Locally Nilpotent Finitary Groups and Associated Rings // Acta Applicandae Mathematicae. 2004. V. 82, № 2. p. 169-181.

16. Levchuk V.M., Suleimanova G.S. Extremal and maximal normal abelian subgroups of a maximal unipotent subgroup in groups of Lie type // Journal of Algebra. 2012. V. 349, № 1. P. 98-116.

17. Левчук В.М. Автоморфизмы унипотентных подгрупп групп Шевалле // Алгебра и Логика. 1990. T. 29. № 3. C. 315-338.

18. Левчук В.М. Автоморфизмы унипотентных подгрупп групп лиева типа малых рангов // Алгебра и Логика. 1990. Т. 29. № 2. С. 141-161.

19. Левчук В.М., Литаврин А.В. Гиперцентральные автоморфизмы нильтреугольных подалгебр алгебр Шевалле // Сиб. электрон. матем. изв. 2016. Т. 13. С. 467-477.

20. Gibbs J.A. Automorphisms of certain unipotent groups // J.Algebra. 1970. V. 14. № 2. P. 203-208.

21. Cao Y., Jiang D., Wang D. Automorphisms of certain nilpotent algebras over commutative rings // International Journal of Algebra and Computation. 2007. V.17, № 3. P. 527-555.

22. Serre J.-P. Algebres de Lie semi-simple complexes, New York - Amsterdam, Benjamin, 1966.

23. Levchuk V.M. Connections between a unitriangular group and certain rings. Part 2. Groups of automorphisms // Siberian Mat. J. 1983. V.24. P.543-557.

24. Левчук В.М., Минакова Е.В. Автоморфизмы и теоретико-модельные вопросы для нильпотентных матричных групп и колец // Фундамент. и прикл. матем. 2008. Т. 14, № 8. С. 159-168.

25. Левчук В.М. Нильтреугольная подалгебра алгебры: обертывающая алгебра, идеалы и автоморфизмы // Доклады Академии наук. 2018. Т. 478, № 2. С. 137-140.

Зотов Игорь Николаевич

Поступила 10.12.2015

ассистент кафедры алгебры и математической логики

Института математики и фундаментальной информатики

Сибирского федерального университета

e-mail: zotovin@rambler.ru.ru

Левчук Владимир Михайлович

д-р физ.-мат. наук, профессор кафедры алгебры и математической логики 
Института математики и фундаментальной информатики

Сибирского федерального университета

e-mail: vlevchuk@sfu-kras.ru

IN. Zotov. Siberian Federal University, Krasnoyarsk, 660041 Russia, e-mail: zotovin@rambler.ru

V. M. Levchuk. Dr. Phys.-Math. Sci., Siberian Federal University, Krasnoyarsk, 660041 Russia, e-mail: vlevchuk@sfu-kras.ru 